\title{
ON THE E/P-COSMIC RAYS AS MEDIATORS OF VISCOUS FORCES THAT CREATE SHOCK AND VORTEX STRUCTURES IN THE RADIO GALAXIES
}

\author{
N.O.Tsvyk \\ Institute of Radio Astronomy of NAS of Ukraine, \\ Chervonopraporna 4, 61002, Kharkiv, Ukraine, natalitsv69@gmail.com
}

\begin{abstract}
There are for the galaxies of FRII type (like Cygnus A) studied a number of hydrodynamic and kinetic processes to transport of magnetized plasma with cosmic rays, and a stratification process of RG-lobe because of elp-cosmic rays. We found that the jet and bow shock in radio galaxies are supported by the processes of elp-cosmic rays (acceleration, radiation), and the effects of changes in viscosity inside the cocoon. Cosmic rays are working inside the "jet head" into the cocoon stratification: to the light caviton (with high kinematic viscosity) and to the heavy shock cover (with high magnetic viscosity). The post-jet-vortex is injected by diffusion of e-rays through the area of "jet head" MHD-turbulized, and it is flowing a caviton around. Alternatively, the bow shock accompanied super sound turbulent waves and flows with p-rays.
\end{abstract}

Keywords: Radio galaxy: cosmic rays, viscosity, shocks

\section{Introduction. A history for the simulation of the FRII-type radio galaxy lobes}

There was in Kaiser (1997) shown a self-consistent hydrodynamical model for the jet-cocoon of FRII radio galaxy (RG), with reconfinement jet-shock (that connected with e-jet plasma production). The other main work, Zanni (2003), show a simple of self-consistent cocoon shock model of FRII RG; and in a work of Kino (2004) was illustrated a hot spot of two-shocks dynamic in details (the parameters of Cygnus A galaxy found).

There are Mathews $(2011,2012)$ recently shown a model for self-consistent evolution of gas and cosmic rays (CRs) in Cygnus A, in assuming a wind-injection of the viscous e-gas with e-CRs into the lobe (cocoon) of Radio Galaxy. This model shows the post-jet-wind with e-CRs; how e-CRs mix with cluster gas; how viscosity supports the e-wind-flow.

In work of Tsvyk (2010) was discussed a model of RG with two-layer cocoon that separated by e- and p-CRs; and this model is developed here. New points in this report: pCRs are the shock-inductors; effects of CRs in turbulence and current production; effect of viscosity changes as flow-regulator. We grounded in main assumptions of Mathews (2012), but show the cocoon dynamics in consist with two simple physical structures: a shock-like-outerlayer and a vortex-flow winding the inner-layer. The aim of this work is to study when the main structures of FRII-
RG (jet, cavity-lobes and bow-shocks) are grown up because of stratification effects in the magnetized plasma through the e $\backslash p$ cosmic rays as mediators.

\section{The main impacts of the CRs to the FRII-lobe}

There key assumptions are: (1) a cocoon matter composed of electron-positron e-plasma (density of $n_{e}=n_{e l}+$ $\left.n_{e 2}\right)$, and ion-proton plasma $\left(n_{p} \approx n_{t h}\right)$, atoms and neutrals $\left(n_{a}\right)$, e- and p-CRs; (2) a cocoon matter is quasi-neutral $\left(n_{e 1^{-}} n_{e 2}=n_{t h}\right)$ but current exists in it; (3) the CRs, that have being accelerated by DSA-mechanism near HS and bow shock, are the main mediator of exchanges due to they relativistic properties; (4) the e- and p-CRs separate in different aria because of flow dynamics and viscosity changes; (5) a magnetic field $(B)$ is produced by regular part from the CRdiffusion currents, and by dynamical part (the jet elongated) from pumping in the frozen of e-flows and from the $\mathrm{p}-\mathrm{CR}$ kinetic effects of mixed matters (mainly within the shock areas). Out the scope are the ions-CRs decays, eannihilation and many other processes.

The main part of cocoon energy contains in p-CRs; and some essential part is in e-CRs too. The less part cocoon energy contains in $B$-field, but frozen effects do $B$-field rule the flow cocoon dynamics. Cocoon energy contains in the p-CRs, the e-CRs, the turbulence (waves and vortexes), the thermal matter and the magnetic field. A jet consists of the e-plasma, with some part of p-plasma; but the p-flows are the most inert and energetically. The eplasma flows are much frozen in B-fields and they fill the inner-cocoon; against to the p-plasma fills the outer cocoon and pumping shocks because of there momentum. Cocoon stratifies because of viscosity changes and injection of Hill's vortex flows (jet-supported). The radiation of e-CRs (synchrotron in radio, IC in X-ray band) helps for e-plasma vortex glues together and separated from $\mathrm{p}$ plasma turbulence flows.

The jet and the lobes parameters of RG are differ in the shock-like (Zanni, 2003) or wind-like (Mathews, 2011) models. For example, RG of Cygnus A located in cluster of $3 \cdot 10^{14}$ sun-mass and with Virial radius of $1 \mathrm{Mpc}$. The jet length is $60 \mathrm{kpc}$, bow-shock velocity is $\sim 6000 \mathrm{~km} / \mathrm{s}$ $(0.02 c)$, and the age of this source is $\sim 10 \mathrm{Myr}$ (it adjusts with the observations of e-CRs radio-age, see Machalski (2007)). So, the shock-like model (Zanni, 2003) predicts the RG jet-power of $L_{j} \sim 10^{47} \mathrm{erg} / \mathrm{s}$ and the energy of $E_{c}=3$. 
$10^{61} \mathrm{erg}$; against to the wind-model Mathews (2012) that give us $L_{j} \sim 10^{46} \mathrm{erg} / \mathrm{s}$ and $E_{c}=3 \cdot 10^{60} \mathrm{erg}$, that predicts only $1 / 20$ part of cocoon volume fills with e-CRs without pCRs energy in this wind-model. We review later shocklike model (illustrated in fig.1), and consider that p-CRs energy is essentially.

Bow shock properties in the outer lobe area. Shockpresser equals to the presser of $\mathrm{p}$-CRs in outer lobe, both and nearly equal to the e-CRs presser in the inner lobe (see fig.1: 2, 3-arias, respectively). Pressure of $B$-field is negligible. The number of $\mathrm{p}-\mathrm{CRs}$ in the outer lobe is some part of p-plasma $(\sim 1-10 \%)$; and it is comparable to the number of e-CRs in this area.

Shock dynamic was shown in Zanni (2003). This a shock scale $R_{s}(t, \theta)$ grows with velocity $u_{s h}$ that defines from the shock presser and the ICM-plasma density, $u_{s h}=$ $d R_{S}(t, \theta) / d t \sim\left[P(t) / \rho_{1}(t)\right]^{1 / 2}\left(\cos ^{2} \theta+a_{1}\right)$, and has an elongation along the jet-direction (for $\theta=0$ we have $1+a_{1} \sim 1.3$ ). The other way, the energy of cocoon defines from the jet burst power (that comes with flow injection of e-CRs plasma), and as well as from shock presser multiplied to cocoon volume, $E_{c}(t) \sim \int L_{j}(t) d t \sim P(t) R_{s}(t, 0)^{3}$. The jet plasma is strongly blown to the inner lobe (3-area in fig.1), and it compresses the cluster matter of outer lobe (1-area, ICM) into the bow-shock front (2-area).

The flow velocities are super-sonic and not regular behind the shock front. Here is the turbulence dominated in plasma dynamics that pumps the Meshkov-vortex instabilities and ejects the inner-lobe-flow vortex surrounding bow-front. The e/p-CRs help to transport shock energy because there high transcalency. The jet-hot-spot, being the shock-center-point, forms the caviton-like feature in density map (see fig.1: 3, 4-arias) which may work to focus the wave-shock energy and support the jet.

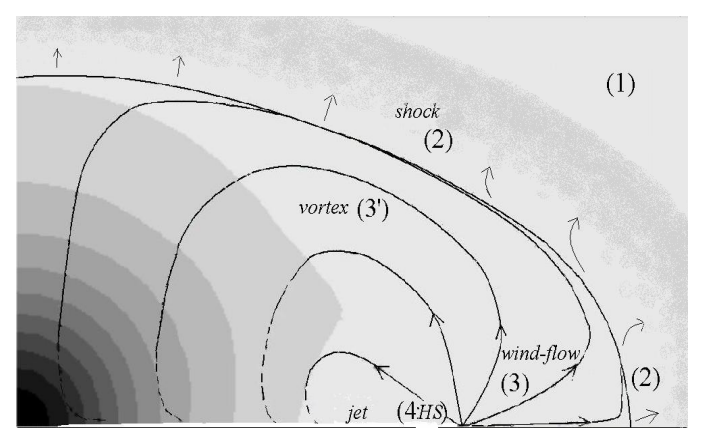

Figure 1: The density field (gray scales) and the velocity field (vector-lines) within the inner (3) and outer lobes (2).

Wind-flow properties in the inner lobe-caviton area. Wind-flow dynamic was shows in Mathews (2011, 2014); the simplest model is "simple-source" injection of e-fluxmatter with e-CRs into the outer-lobe ep-plasma (with some part of neutral atoms). It illustrated in fig. 1 see aria 3 ; and the model of parameters done in Tables 1,2 . The pressure $(P)$ and flow velocity $(u)$ make the dynamical variations such as in the injection point (hot spot at jethead) we have $P \rightarrow 0$ and $u$-velocity is maximum. The average flow velocity consists with regular and diffusion parts, the density varies with velocity profile, as $\rho \propto r_{s}^{q-2}$ when $u \propto(c / 3) r_{s}^{-q},\left(q \leq 2, r_{s}\right.$ is a scale from HS to the chosen point). It is likely that post-jet e-plasma wind to the lobe (3) as nearly regular flow together with e-CRs and some part of thermal plasma from area (2), because this matters have mixed gradually within the HS-area and accompanied the accelerations of the e-CRs. That way, in the injection HS-point we have a purely post-jet flow with the density as in Kino (2004) model (see Table 1); and then the post-jet flows mix with $\sim 0.2 n_{t h}(r)$ of outer thermal plasma because of e-CR diffusion (see Table $2 ; r$ is a scale from RG-center to the chosen point, a density derives in the King's model as $\left.n_{t h}(r) \propto\left(1+r^{2} / a_{K}^{2}\right)^{-1.13}\right)$. This edominated-flow becomes regular because of viscosity in wind-injection area. Latter, in outside, the flux-collisionfront give us the maximum of presser, that exhibit as $u=0$ and thermal-heats dominate. In this flow-stress cover, where the wind-flow transforms into the macro-scale Hill's-2 vortex, we have: $\rho_{e} u \sim \rho_{t h} u_{2}, u_{2} \approx 0.75 u_{s h}$; and the matters are mixed strongly by vortex-turbulence and MHD-waves, as e-CRs pressure is comparable to thermal pressure, $P_{t h} \geq P_{e C R}$. So, the wind-velocities and Hill's vortex velocity are correlated here.

Table 1: The parameters of Cygnus A at near HS-area based on Kino (2004) model: the density ( $\rho$ and $n_{a}, n_{p}, n_{\mathrm{e}}$ ), magnetic field $(B)$, scattering time $(\tau)$, viscosities $\left(\eta_{K}, v_{K}\right.$, $\left.v_{m}\right)$, collision $\left(\mathrm{X}=\omega_{B} \tau, \beta \sim 0.3\right)$ and Reynolds $(R e)$ numbers.

\begin{tabular}{|c|c|c|c|c|}
\hline & 1-ICM & 2-HS & 3-HS & 4-jet \\
\hline \begin{tabular}{|l|} 
Presser, \\
${\text { dyne } / \mathrm{cm}^{2}}^{2}$
\end{tabular} & $10^{-10}$ & \multicolumn{2}{|c|}{$\frac{1}{210^{-8}}$} & $<10^{-8}$ \\
\hline$n_{a} / n_{p}$ & 0.20 & 0.05 & $<0.01$ & 0 \\
\hline$n_{p} ; \mathrm{cm}^{-3}$, & 0.025 & 0.1 & $10^{-5}$ & $10^{-7}$ \\
\hline$n_{e}, \mathrm{~cm}^{-3}$ & 0.025 & 0.1 & $10^{-3}$ & $10^{-4}$ \\
\hline$B, \mathrm{G}$ & $10^{-5}$ & $7 \cdot 10^{-5}$ & $2 \cdot 10^{-4}$ & $2 \cdot 10^{-5}$ \\
\hline$\rho, m_{p} / \mathrm{cm}^{3}$ & 0.03 & 0.1 & $10^{-5}$ & $6 \cdot 10^{-7}$ \\
\hline$\omega_{B} \tau_{i i},\left[\omega_{B e} \tau_{e e}\right]$ & $10^{11}$ & {$\left[10^{14}\right]$} & {$\left[10^{20}\right]$} & {$\left[10^{19}\right]$} \\
\hline$\tau_{e i},\left[\tau_{e e}\right], \mathrm{yr}$ & $10^{5}$ & $10^{7}$ & {$\left[10^{12}\right]$} & {$\left[10^{12}\right]$} \\
\hline $\begin{array}{l}\eta_{K}, \mathrm{gm} /(\mathrm{s} \mathrm{cm}) \\
(\beta=0.3)\end{array}$ & $5 \cdot 10^{-5}$ & 0.25 & 35 & 10 \\
\hline$v_{K},(\beta=0 . .0 .3)$ & $10^{25 . .21}$ & $10^{29 . .24}$ & $10^{37.30}$ & $10^{36 . .31}$ \\
\hline$v_{m}, \mathrm{~cm}^{2} / \mathrm{s}$ & $10^{21}$ & $10^{20}$ & $10^{14}$ & $10^{6}$ \\
\hline $\begin{array}{l}R e=\lambda_{0} c / v_{K} \\
\left(\beta=0.3, \lambda_{0} \sim 1 \mathrm{kpc}\right)\end{array}$ & $10^{11}$ & $5 \cdot 10^{7}$ & 50 & 10 \\
\hline
\end{tabular}

Vortex properties in the inner lobe-caviton area. Some of the vortex-flow dynamic with vortex-injection was shows in Antonuccio-Delogu (2010). We add this sketch of processes some more connection with shockstructures. This way, the main vortex is injected by Carioles force in around of min-pressure-focus $(P \rightarrow 0)$ that forms as a ring near the hot spot points because of fluxstress dynamic in the post-jet structures of e-flux-matter blows. The injection provides within Kelvin's low frames: $d(\vec{\omega} / \rho) / d t=(\vec{\omega} / \rho) \nabla \vec{u} \quad(\omega$-circulation is constant, $\vec{\omega}=\operatorname{rot} \vec{u})$. And the gravity-buoyant and $F_{\eta}$-viscosity forces help us to form the vortex flux of Hill's-2 like type 
in the post-jet cavity surface around (see fig.1, 3' area), because of the matter difference and e-CR presser variation:

$$
\frac{\partial \vec{u}}{\partial t}+\rho^{-1} \nabla P+\frac{1}{2} \nabla\left(u^{2}\right)=[\vec{u} \times \vec{\omega}]+\vec{F}_{\eta}+\vec{F}_{g} .
$$

A simple model of Hill's-2 vortex of $R_{s 3}$ size (limited of e-plasma contact border, $R_{s 3} \sim 0.7 R_{s}$ ) may describe as main-flow-vortex in nearly uncompressed matter by the velocity profile in spherical $(r-\theta)$ geometry with the RG centre as zero-point and jet aligns to $\theta=0$ :

$$
\begin{aligned}
& u_{r} \propto \rho_{t h}(r)^{-1}\left(R_{s 3}(t, \theta)^{2}-r^{2}\right) \cos \theta \cos (2 \theta) ; \\
& u_{\theta} \propto \rho_{t h}(r)^{-1}\left(R_{s 3}(t, \theta)^{2}-r^{2}\right) \cos \theta \sin (2 \theta) .
\end{aligned}
$$

A fine density variation is accounted as $u \propto \rho_{t h}(r)^{-1}$ in attached to the King's model. This main-vortex excites the vortex-turbulence in contact with shock-surface that runs plasma density to mix by everyone small or large flowvelocity lines. The turbulence makes to shift dynamically this Hill's vortex forward together with bow-shock surface. The plasma presser inside the any macroscopic flow tube is decrease because the e-CRs are radiated, and this supports the Carioles force in Hill's-2 vortex structure.

Vortex flux matter composed with e-plasma and e-CRs that predominates over the thermal ep-plasma in the inner cocoon part $\left(n_{e}>n_{t h}\right)$. This forms a cavity-vortex structure within the inner e-Lobe density field, $\rho=\left(n_{t h}+n_{a}\right) m_{p}+n_{e} m_{e}$, and the Hill-2 vortex injection helps to separate the innermatters with e-CRs from outer-matters with p-CRs. The particles are sorted by quasi-neutrality low $\left(n_{e 1^{-}} n_{e 2}=n_{t h}\right)$.

The average flux velocity $(u)$ obtains as to dominate the e-plasma flux and the relativistic e-CR diffusion flux: $u=$ $\left(\sum n_{\alpha} m_{\alpha} u_{\alpha}\right) / \rho$ ( $\alpha$ is types of particles: $(e 1, e 2)-$ electron/ positrons, $p$ - protons, $a-$ neutral atoms, and $e l p-C R-$ relativistic particles). Superposition of the vortex and the shock velocity gives up the flux relaxation $(u \rightarrow 0)$ at $\theta \sim \pi / 2$. Near the e-lobe edge ( $R_{s 3}$-surface) we have $u \sim u_{2}$; and in this area the matter composition changes: the eplasma is depressed and the thermal plasma with $\mathrm{p}-\mathrm{CRs}$ is added, whit the presser is conserved.

To construct the density model (fig. 1, Table 2) we use the continuity matter equation with referred assumptions and with $u$-flux model chosen.

\section{Magnetic fields impact to FRII-lobe structure}

The magnetic field is grown both the dynamical and regular field parts inside the cocoon. The dynamical field is pumping from cosmic turbulence $B_{\text {turb }}$ field by adiabatic and alpha-pump turbulent effects; the regular part of $B_{0^{-}}$ field induced from CR-diffusion currents.

Only estimation lobe model parameters are represented in Table 2, and the $B$-field calculations in this model show in fig.2. If the current is estimated in the inner lobe because of diffusion motion of the e-CR-particles with the different singles, $\Delta n_{e} \sim \Delta n_{C R} \sim 0.1 n_{t h}$ (despite of $n_{e}>>n_{t h}$ in the 3-area) and $\Delta v \sim\left|v_{\mathrm{e}}-u\right| \sim<v_{\text {dif }}>\sim 10^{6} \mathrm{~cm} / \mathrm{s}$ (that is smaller then sound and Alfvenic velocities, $c_{s, A} \sim 10^{7} . .10^{9}$ $\mathrm{cm} / \mathrm{s}$ ), so the regular part of magnetic field give us tor- oidal-field of $B_{0} \sim 3 \cdot 10^{-4} \mathrm{G}$ that mainly induced by radial and jet-elongate current components.

Beside this, the $\mathrm{p}$-CRs diffusion give us a moves $B$ field lines together with cosmic matter, and pumps dynamically the turbulent field component as alpha-pump turbulent effects in (2) and HS areas (fig.1). These effects are dominated within the outer lobe that is near the bow shock-wave surface; we assume that $B_{\text {turb }} \propto\left(P_{e C R}+P_{p C R}\right)$. Stress of these turbulent fields must be $B_{\text {turb }} \sim 3 \cdot 10^{-4} \mathrm{G}$, compare to the regular $B_{0}$-field near the jet-HS surface.

Maximum strength of regular magnetic field $\left(B_{0}\right)$ is nearly denoted to the areas of minimum plasma presser (the same as areas of with minimum of Hill's-flow velocities). This effect comes to gluing of local e-flows with eCRs, and it may appear as bright features (look likes as an additional HS-spot) in radio emission map of the RG-lobe. However the turbulent pumping may be so strong that to exhibit only one HS-point in the lobe map as the jet head.

Table 2: The model parameters of inner and outer lobe for typical FRII-RG (like Cygnus A) based on assumption of

\begin{tabular}{|c|c|c|c|c|}
\hline AREA & $\begin{array}{c}3 \\
(\mathrm{HS})\end{array}$ & $\begin{array}{c}2 \\
(\mathrm{HS})\end{array}$ & $\begin{array}{c}3 \\
\text { (e-Lobe) }\end{array}$ & $\begin{array}{c}2 \\
\text { (outer) }\end{array}$ \\
\hline$n_{e}$ & 0.001 & 0.0005 & $10^{-4}$ & $<10^{-4}$ \\
\hline$n_{t h}, \mathrm{~cm}^{-3}$ & 0.001 & 0.04 & 0.002 & 0.003 \\
\hline$n_{a}$ & $10^{-6}$ & 0.0003 & $<10^{-4}$ & $<10^{-4}$ \\
\hline$B_{0}, m G$ & $0.15 . .0 .2$ & 0.03 & $0.3 \ldots 0.2$ & 0.06 \\
\hline$\Lambda_{l l} .$. & $0.1 .$. & $0.1 .$. & 0.5 .. & 10 .. \\
\hline$\Lambda_{r}, k p c$ & $10^{-5}$ & $10^{-6}$ & 0.1 & 0.5 \\
\hline$v_{\mathrm{m}}$ & $10^{13}$ & $10^{15}$ & $10^{18}$ & $10^{19}$ \\
\hline$v_{\mathrm{K}}^{*}$ & $10^{35}$ & $10^{31}$ & $10^{13}$ & $10^{20}$ \\
\hline$R e=\lambda_{0} \mathrm{c} / \nu_{\mathrm{K}} *$ & $\begin{array}{c}<1 \\
\text { (reg. flow) }\end{array}$ & $\sim 2$ & $\begin{array}{c}10^{7} . .10^{20} \\
\text { (turb.) }\end{array}$ & $\sim 10^{13}$ \\
\hline $\begin{array}{l}\lambda_{\min }, \mathrm{pc} \\
(\operatorname{Re} \sim 1000)\end{array}$ & $>10000$ & $>1$ & $<10^{-6}$ & $<0.1$ \\
\hline
\end{tabular}
the wind-vortex-shock cocoon transformation because of the CR-flow dynamics (see the text and Table 1 in detail).

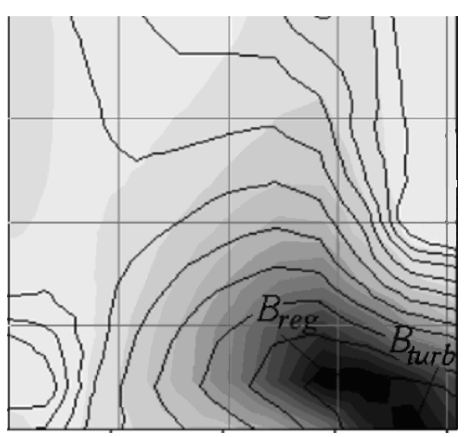

Figure 2: The model of magnetic field strength (in the gray scales: dark is $B \sim 3 \cdot 10^{-4} \mathrm{G}$, white is $B \sim 10^{-6} \mathrm{G}$ ) and the low-band synchrotron radiation (contour lines). 


\section{Model for the RG lobe viscosity as cosmic plasma}

Cosmic plasma is low-collision matter (the collision term is $X_{\alpha}=\omega_{B \alpha} \tau_{\alpha}>>1$, and the magnetic presser is smaller then thermal one $P_{B}<P_{n T}$ ), and this plasma is composed of the different types $(\alpha)$ of particles, that parameters are changed within the RG-cocoon.

The contact layer that splitting the inner lobe (3) with e-plasma and the outer lobe (2) with thermal plasma plus $\mathrm{p}-\mathrm{CRs}$, is the front of viscosity stress that comes to change the matters dynamics. Viscosity consists of kinematical and magnetic parts, and the physics of cocoon are coordinated by these types of viscous vary.

This way, the kinematical viscosity $\eta_{K}$ coordinates the changes of the velocity along $B_{0}$ (by $\eta_{K 1}$ ), and the viscous dump of transverse velocity fluctuations (by $\eta_{K} \sim \eta_{K 0}$ ). Here we are: $\eta_{K 1} \sim \eta_{K 0} X_{\mathrm{j}}^{-2+\beta}$, where $\left.\eta_{K 0}=\Sigma\left\{n_{j} T_{j} \tau_{j} X_{j}^{-\beta}\right)\right\}$ and $0<\beta<1$ is the magnetic turbulence parameter. The $\mathrm{p}$ CRs make to change the characteristics $X$ and $\beta$ of the slow matter turbulence that handle the viscous forces.

The magnetic viscosity, $\eta_{m}=\rho v_{m}=\rho c^{2} / \sigma_{e f f}$ coordinates the currents induction and the enhanced of MHDturbulence within the shock both and in the jet surface; both and it enhanced the magnetic dynamo in the dense matter area. Here is the effective conductivity within elobe depends on the neutral density $\sigma_{e f f} \sim e^{2} n_{e} \tau_{e} m_{e}^{-1}(1+$ $\left.\left(n_{a} m_{p} \rho^{-1}\right)^{2} X_{\mathrm{e}} X_{\mathrm{i}}\right)$ as accounted in Bykov (2007) model.

Table 1 and 2 demonstrate the typical parameters in cocoon as changes of the kinematical and the magnetic viscosity, both and as turbulent exiting changes (by Reynolds number). The fig.3 demonstrates the relative viscosity changes of $v_{m} / v_{K}$ that response the areas where magnetic turbulence may pump strongly.

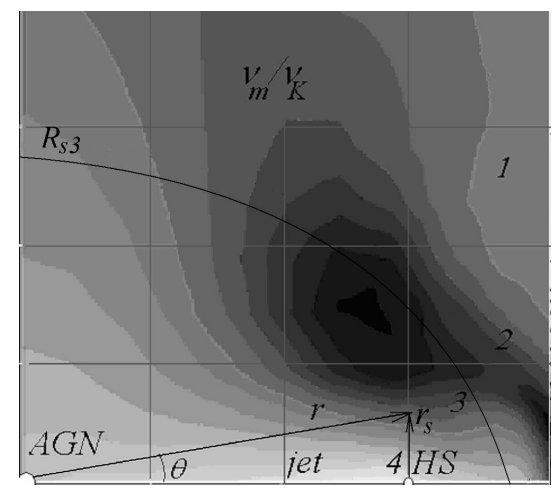

Figure 3: The model of relative viscosity changes (in the gray scales: dark is $v_{\boldsymbol{m}} / v_{\boldsymbol{K}} \sim 10^{10}$, white is $v_{\boldsymbol{m}} / \boldsymbol{v}_{\boldsymbol{K}} \sim 10^{-20}$; the 2-3 contact $R_{s 3}$-boundary outline schematically sketched).

\section{Radiation and streaming of the e-CRs in FRII lobe}

In the inner lobe, the wind-flow consists of the local flows with e-CRs which are gluing because of viscosity, magnetize effects and radiating cooling of e-CRs. The diffusion effect of the e-CRs, because of vortex and Alfven-wave turbulence, are smoothing the flow dynamic and dumping turbulence by viscosity driven. The effect of e-CRs radiation leads to cool of the lobe matter and it helps a current production and gluing of local e-CR-flows.
The e-CRs are radiating mainly by synchrotron (in radio bands at $f$-frequency) and synchrotron-inverse Compton (in X-ray bands) processes, which are frequencydependent. So, the lobe radio brightness is $I_{s y n} \propto n_{e C R} B$ $f^{(-s+1) / 2}$, and the X-ray brightness depends on $n_{e C R}$ and radiation field.

The elp-CRs flow together with the thermal plasma (that frozen in $B$-field tubes) and drift with energydependent diffusing velocity, $v_{e C R}=u+v_{\text {diffeCR }}$. A diffusion velocity defines by turbulence parameters and regular magnetic field $\left(B_{0}\right): v_{\text {diffeCR }} \sim\left(\kappa_{e 0} / \Lambda_{\text {diff }}\right) \gamma^{2-s} \propto\left(B_{0} / B_{\text {turb }}\right)^{2} r_{g}$ $c / \Lambda_{\text {synn }}$. Thus, the low-energy particles of e-CRs, that was cooling by radiation to energy $\gamma \sim 1 . .10$, have to flow with thermal plasma better then diffuse. Alternatively, the pCRs particles have to correct a local $B_{\text {turb }}$-lines with any diffusion act, thus in a bow shock area this effects are limited the magnetic field correlation scales $\left(\lambda_{0}\right)$ with the pCRs energy spectrum $\left(\gamma_{p_{-} \max }\right)$ and with $R_{s}(t)$ lobe-size.

This way, taking account the trends of B-field and eflows, we may predicted that a powerful radio emission of low-energy e-CRs in $\mathrm{MHz}$-band comes from areas where e-rays are pulled together into streams, due to the turbulence by viscous forces dumping, and e-rays cooling. And the radiation-cooling of e-CRs give us the main mediatoreffects that coordinate all cocoon dynamics.

\section{Conclusions}

1. The viscosity of RG matter rules in the lobephenomenology. It is switching the instabilities, and directing the gluing flows, and heating or accelerating of CRs in the cocoon, and the magnetic-viscosity divides the vortexes into small modes ( $B$-pumping).

2. The bow-shock transports by wave-turbulence, and by the p-CRs, that getting up of magnetic-viscosity the biggest. The elp-CRs ejects by diffusion-shock acceleration mechanism.

3. The magnetic field turbulence prevents the mixing of cocoon fluid. The e-CRs frozen into magnetic turbulence critically, beginning with $>10^{-6}$ pc-size modes (the smallest). The p-CRs help to mix the matters.

4. The e-CRs and p-CRs are separated by injection of Hill's-2 vortex from relativistic post-jet e-plasma-flow winding. The ties of e-CRs are gluing by viscosity and radiate-cooling effects.

5. The e-CRs diffuse (in adding to flow-transport) by energy-dependent, because of B-turbulence that has been generated by vortexes and MHD-waves.

After synchrotron-radiate-cooling, the low-energy e-CRs are filling nearly all cocoon-volume, adding to the p-CRs that holding up the $B$-turbulence within the cocoon-surface.

\section{References}

Kaiser C.R. et al.: 1997, MNRAS, 286, 215.

Zanni C. et al.: 2003, $A \& A, \mathbf{4 0 2}, 949$.

Kino M. et al.: 2004, MNRAS, 349, 336.

Mathews W.G. et al.: 2011, ApJ, 736, 6.

Mathews W.G. et al.: 2012, ApJ, 755, 13.

Tsvyk N.: 2010, in: Proc. of $10^{\text {th }}$ Gamow's Odessa Astron.

Conf., Odessa, Ukraine, 142.

Machalski J. et al.: 2007, $A \& A, \mathbf{4 6 2}, 43$.

Antonuccio-Delogu V. et al.: 2010, MNRAS, 405, 1303.

Bykov A.M. et al.: 2007, Physics-Uspekhi, 50, 141. 